山्山FFRANÇAISE

$>\mathrm{DE}$

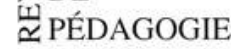

\section{Revue française de pédagogie}

Recherches en éducation

174 | janvier-mars 2011

Évaluation, développement professionnel et organisation scolaire

\title{
Le développement professionnel des enseignants et ses effets positifs sur les apprentissages des élèves
}

Teachers' professional development and its positive effects on student learning

El desarrollo profesional de los docentes y los efectos positivos en los

aprendizajes de los alumnos

Persönliche Entwicklung der Lehrer und positive Auswirkungen auf die Schüler

Helen Timperley

\section{(2) OpenEdition}

Journals

\section{Édition électronique}

URL : http://journals.openedition.org/rfp/2910

DOI : $10.4000 /$ rfp.2910

ISSN : 2105-2913

Éditeur

ENS Éditions

Édition imprimée

Date de publication : 15 mars 2011

Pagination : $31-40$

ISBN : 978-2-7342-1203-4

ISSN : 0556-7807

\section{Référence électronique}

Helen Timperley, «Le développement professionnel des enseignants et ses effets positifs sur les apprentissages des élèves », Revue française de pédagogie [En ligne], 174 | janvier-mars 2011, mis en ligne le 15 mars 2015, consulté le 19 avril 2019. URL : http://journals.openedition.org/rfp/2910 ; DOI : $10.4000 / \mathrm{rfp} .2910$ 


\section{Le développement professionnel des enseignants et ses effets positifs sur les apprentissages des élèves}

Helen Timperley

En Nouvelle-Zélande, une attention grandissante a été portée par les décideurs politiques au développement professionnel des enseignants et à la façon dont il améliorait les résultats des élèves. Cette approche permet de développer des pratiques d'enseignement collaboratives, en plaçant les enseignants dans une position d'enquêteur de leurs propres pratiques. Les programmes de développement professionnel ont des effets réels sur l'amélioration de la réussite des élèves dans le domaine de la littératie, notamment en lecture. Ils permettent aux enseignants de mieux identifier leurs besoins de formation à travers une analyse des difficultés des élèves et la mise en œuvre de procédures d'évaluation efficaces.

Descripteurs (TESE) : formation continue des enseignants, enquête, travail en équipe, évaluation des étudiants, processus d'apprentissage, Nouvelle-Zélande.

\section{INTRODUCTION}

Chaque jour, les enseignants sont confrontés aux changements qu'impliquent les nouveaux programmes, les nouvelles méthodes d'évaluation et les nouvelles technologies. Ils doivent aussi faire face à des publics hétérogènes parfois rétifs aux pratiques pédagogiques les plus courantes. De nombreux pays anglo-saxons ont investi massivement dans le développement professionnel continu des enseignants déjà titularisés pour les aider à relever les défis actuels. Dans certains pays anglophones, les méta-analyses sur les facteurs favorisant les apprentissages des élèves expliquent en grande partie ce changement de cap. Ces analyses montrent invariablement que, parmi tous les acteurs du système éducatif, ce sont les enseignants qui ont le plus d'influence sur les résultats des élèves. Les études proviennent de divers pays, dont l'Australie (Cuttance, 1998), les États-Unis (Nye, Konstantanopoulos \& Hedges, 2004) et le RoyaumeUni (Muijs \& Reynolds, 2001). Si tous reconnaissent que les parents et la communauté éducative jouent un rôle important, ils observent que les enseignants sont le premier levier du changement au sein du système éducatif. 
Malheureusement, l'espoir de voir l'investissement dans le développement professionnel des enseignants entraîner une hausse des résultats des élèves ne s'est pas concrétisé. Dans sa majorité, la littérature de recherche fait état de résultats variables et souvent décevants, à l'origine d'une frustration considérable des observateurs politiques (National Staff Development Council, 2001 ; Sparks, 2004). La variété des résultats montre que certaines méthodes sont plus efficaces que d'autres. J'ai animé une équipe de chercheurs dont la mission était de trouver dans la littérature anglo-saxonne les méthodes d'apprentissage ayant fait leurs preuves, à partir de la question suivante: "Quels types de développement professionnel contribuent à l'amélioration des résultats des élèves ? " (Timperley, Wilson, Barrar et al., 2007) La littérature socioculturelle et psychologique sur le développement professionnel (voir par exemple Donovan, Bransford \& Pellegrino, 1999) nous a permis de construire un cadre d'analyse des études empiriques contribuant aux bons résultats des élèves sur le plan scolaire, comme à leur épanouissement sur les plans personnel et social.

Cet article décrit l'approche retenue pour la synthèse de la littérature et ses résultats principaux. II montre aussi comment les principes et processus reconnus comme efficaces pour le développement professionnel ont conduit à un projet de formation en littératie dans plus de 300 écoles de NouvelleZélande. Les élèves ont progressé deux fois plus que prévu en lecture et en expression écrite au cours des deux années du projet. Les élèves se situant parmi les $20 \%$ les plus faibles ont fait trois ou quatre fois plus de progrès qu'escompté (Timperley \& Parr, 2009b).

\section{APPROCHE MÉTHODOLOGIQUE}

II s'agissait de repérer, dans la littérature, les aspects de la formation professionnelle des enseignants qui, selon divers auteurs, contribuent à l'amélioration des résultats des élèves, pris dans leur diversité. Quatre-vingt-quatre items différents ont été introduits dans un cadre analytique pluridisciplinaire. Ce cadre a servi ensuite à analyser la littérature empirique pour repérer quels types de connaissances et quelles conditions de développement de celles-ci étaient à même de promouvoir les processus d'apprentissage professionnel permettant aux enseignants d'adapter leurs pratiques, et in fine d'avoir un effet positif sur les résultats des élèves. Les items ont été regroupés dans les catégories suivantes : environnement socio-économique au sens large (éducation formelle et politiques curriculaires entre autres), contexte de formation professionnelle (située dans les établissements scolaires ou les universités), contenu de l'offre de formation professionnelle, activités développées pour promouvoir la formation (implication dans des ateliers, tutorat), processus d'apprentissage pour les enseignants, leurs réactions à ces expériences, et enfin impact sur les apprenants (voir l'annexe 1).

Toutes les catégories du cadre d'analyse ont reçu le même traitement en termes de codage de la littérature empirique. Chaque item dans chaque catégorie était traité de façon neutre quant à son impact sur les enseignants ou les élèves, jusqu'à ce qu'il soit mis à l'épreuve de la réalité des faits. À cette fin, une recherche itérative de la littérature internationale a été entreprise à partir de recherches de bases de données électroniques, de l'examen des revues et manuels scientifiques pertinents et d'un réseau d'informateurs, afin de repérer les études qui associaient formation professionnelle des enseignants et résultats des élèves. L'application de critères méthodologiques courants a permis d'identifier 73 études d'interventions indépendantes pour un total de 227 effets (Cohen, 1988). Étant donné les difficultés à trouver des équivalences dans les tailles d'effet ${ }^{1}$, les élèves ont été traités par catégorie et n'ont pas été classés comme il est de coutume dans les méta-analyses. À partir de ces études empiriques, les attributs de la formation professionnelle ont ensuite été cartographiés au sein du cadre théorique, pour repérer quels types de connaissances ont été bénéfiques pour les élèves et dans quelles circonstances elles ont été acquises.

\section{IMPACT DU DÉVELOPPEMENT PROFESSIONNEL SUR LES RÉSULTATS DES ÉLÈVES}

L'analyse de ces études montre que les stages de développement professionnel pour enseignants ont eus pour la plupart un impact positif sur les résultats des élèves. Certes un biais existe : on publie plus facilement les résultats positifs que négatifs. Les résultats concernant les progrès scolaires des élèves présentaient des scores plus élevés que les résultats concernant leurs autres caractéristiques personnelles ou sociales (bien-être, motivation, etc.). Les tailles 
d'effet moyennes, élevées en littératie, s'expliquent par la prise en compte de plusieurs études focalisées sur les élèves à besoins spécifiques, dont le point de départ (niveau plus bas) débouchait sur des effets très élevés. En sciences, ces effets étaient influencés par le grand nombre d'études construites sur des évaluations non standardisées mises au point par des chercheurs. Sans surprise, les études réalisées sur les groupes de contrôle avaient des effets inférieurs à celles réalisées sur les groupes de comparaison, notamment lorsque des instruments standardisés étaient utilisés. Les raisons de ces effets limités sont brièvement décrites ici. Nous y ajoutons une description plus détaillée des conditions nécessaires à une amélioration des résultats des élèves.

\section{Développement professionnel dont l'impact sur les résultats des élèves est limité}

Les circonstances dans lesquelles le développement professionnel était apparemment le moins efficace reflétaient des pratiques opposées. À une extrémité, les enseignants sont considérés comme des professionnels autorégulateurs qui, lorsqu'on leur donne suffisamment de temps et de ressources, parviennent à construire leurs propres expériences d'apprentissage et assurent un enseignement plus efficace grâce à leur expertise collective. Nous n'avons malheureusement trouvé guère de preuves à l'appui de cette assertion ; le constat indiquait plutôt l'inverse (Lipman, 1997 ; Saxe, Gearhart \& Nasir, 2001). En fait, cette méthode n'approfondissait pas les connaissances et pratiques des enseignants, pas plus qu'elle ne remettait en cause les attitudes problématiques. À l'autre extrémité, des experts extérieurs donnaient des « recettes » pour enseigner (souvent fondées sur des recherches présentant un type de pédagogie efficace pour améliorer les résultats des élèves), puis présentaient des pratiques toutes faites aux enseignants, avant de s'assurer du suivi à la lettre de l'application de ces recettes (Borman, Slavin, Cheung et al., 2005 ; Sijde, 1989). Cette méthode peut s'avérer plus efficace et permettre d'améliorer davantage les résultats des élèves que celle consistant à laisser les enseignants trouver leurs propres solutions. Ses résultats restent néanmoins précaires ou relativement limités par rapport à d'autres types de formation professionnelle (Borman, Slavin, Cheung et al., 2005 ; Datnow, Borman, Stringfield et al., 2003).

\section{Développement professionnel contribuant à l'amélioration des résultats des élèves}

Dans cette partie, nous allons aborder le contenu de l'offre de formation professionnelle, les activités construites pour encourager l'apprentissage des enseignants et les processus d'apprentissage des élèves.

\section{Le contenu de l'offre de formation}

Les situations dans lesquelles les gains étaient les plus élevés (concernant la compréhension écrite par exemple) portaient sur l'acquisition par l'élève de nouvelles connaissances et compétences, indépendamment de son environnement socioculturel. Les acquis d'apprentissage des élèves faisaient la différence. Ces apports devaient être aisément exploitables pour ensuite déterminer les choix pédagogiques des enseignants. Ces grandes tendances étaient valables aussi bien pour l'enseignement primaire que pour le secondaire. Les savoirs pédagogiques s'entendent au sens qu'en donne Shulman (1986, 1987), à savoir des contenus spécialisés associant connaissances universitaires et pédagogiques. Ces contenus permettent aux enseignants « de transformer leurs savoirs pour rendre leurs pratiques pédagogiques efficaces tout en les adaptant aux capacités et aux acquis différents des élèves " (Shulman, 1987, p. 15). Les savoirs sur l'évaluation s'entendent par la capacité des enseignants à faire le diagnostic des besoins d'apprentissage des élèves, de telle sorte que leur enseignement puisse être en phase avec les acquis des élèves. Ces savoirs sur les évaluations renseignent aussi les enseignants sur les progrès à accomplir s'ils veulent avancer dans l'enseignement du programme. Ainsi une formation professionnelle efficace se caractérise entre autres par une connaissance de l'intérêt et des limites de tel ou tel test, mais aussi par une utilisation d'évaluations plus informelles. Aucun de ces deux types de savoirs ne se suffit à lui-même, mais ils doivent être intégrés dans une théorie de l'apprentissage cohérente. Les enseignants enseignent de manière holistique à partir de leurs convictions sur les élèves et de la façon dont ils apprennent, mais également à partir de l'idée qu'ils se font de la valeur de chaque programme et des meilleures façons de l'enseigner.

Les formations concernant l'acquisition de savoirs isolés et indépendants d'une théorie cohérente et efficace de l'apprentissage ne sont utiles qu'au développement de compétences également isolées, telles que la connaissance des phonèmes (Baker \& Smith, 1999) ou l'apprentissage de la lecture de 
cartes (Fishman, Marx, Besta et al., 2003). Une formation plus générale sur la façon d'enseigner la compréhension écrite ou le raisonnement scientifique est beaucoup plus exigeante. L'intégration de ces apprentissages s'explique par le fait que les informations issues de l'évaluation diagnostique permettent aux enseignants de comprendre en détail les acquis des élèves et leurs besoins. Les connaissances acquises et les contenus à évaluer ne se limitent pas aux résultats des tests : ils comprennent aussi l'observation fine de l'apprentissage des élèves, de leur façon de travailler, ainsi que des entretiens avec les élèves sur leur maîtrise de certains concepts. La connaissance de ce que les élèves savent faire ou non est utile pour ajuster les pratiques de cours vers un meilleur apprentissage des élèves, mais seulement si les enseignants ont une maîtrise suffisante des savoirs pédagogiques sur lesquels ils peuvent fonder leurs choix. Sinon l'analyse des évaluations demeure superficielle et ne permet donc pas d'en tirer profit. Toutes les études où les tailles d'effet étaient élevées mettaient l'accent sur l'accompagnement des enseignants pour transférer les savoirs pédagogiques et évaluatifs dans leurs pratiques. Ce transfert n'était pas laissé au hasard. Des messages décontextualisés plaqués sur des pratiques innovantes n'avaient en définitive aucun impact sur les élèves.

\section{Les activités bénéfiques aux apprentissages}

Une grande partie de la littérature sur le développement professionnel des enseignants porte sur les activités, notamment les ateliers ou le tutorat pour enseignants, plutôt que sur les contenus enseignés. Dans les faits, aucune activité en particulier ne garantit de meilleurs résultats qu'une autre. Toutes ont des effets à la fois positifs et limités. Par exemple, un cours est efficace lorsque les enseignants comprennent la théorie qui sous-tend sa pratique. Ce qui compte plus que la forme d'une activité, c'est de déterminer si elle permet d'aider les enseignants à acquérir à la fois les connaissances et compétences pertinentes et la façon dont ils peuvent transférer ces connaissances dans leurs pratiques de classe.

\section{Les processus d'apprentissage}

Dans l'annexe 1, l'autre catégorie qui indique un impact significatif sur l'amélioration éventuelle des résultats des élèves concerne les processus d'apprentissage des enseignants. Les études empiriques qui ont abordé directement cette question sont rares mais elles ont montré l'importance du phénomène. Pour la plupart des formateurs, il suffit que les ensei- gnants intègrent les nouvelles informations ou les compétences pédagogiques qu'ils leur apportent pour rendre leurs pratiques plus efficaces. Ce processus ne fonctionne que si ces nouvelles pratiques sont en phase avec les convictions et les hypothèses qui sous-tendent leurs pratiques habituelles. Cette méthode fonctionne également lorsque les enseignants sont conscients que leurs savoirs et compétences pédagogiques sont limités et qu'ils cherchent activement à les enrichir. Ce cas est néanmoins assez rare, dans la mesure où la formation professionnelle concerne des enseignants titularisés qui se considèrent compétents. II en faut beaucoup plus pour changer les pratiques lorsqu'elles ne sont pas aussi efficaces que ce que les enseignants pensent. Dans ces circonstances, il faut que les enseignants comprennent la différence entre leurs pratiques actuelles et les changements à opérer. De nouveaux apprentissages peuvent contribuer à ébranler leurs convictions les plus ancrées, notamment si on leur montre qu'une nouvelle manière d'enseigner peut améliorer les résultats des élèves. La question de savoir ce qui fait un bon programme et comment il doit être enseigné, surtout en mathématiques et en sciences, a besoin d'être repensée pour que les enseignants passent de l'acquisition de faits à leur utilisation pour résoudre des problèmes. Dès lors, l'enjeu est de motiver les enseignants pour qu'ils acquièrent de nouvelles connaissances professionnelles. Néanmoins, comme tous les apprenants, s'ils sentent que leur conduite est dictée par un tiers, ils ont toutes les chances de résister.

Les études les plus efficaces sont celles où les enseignants deviennent co-apprenants ou apprenants autorégulés par un processus d'enquête situé dans l'environnement immédiat de leur pratique (voir par exemple Carpenter, Fennema, Peterson et al., 1989 ; McNaughton, Lai, MacDonald et al., 2004 ; Timperley \& Parr, 2009a). Les enseignants doivent identifier et faire le diagnostic des problèmes d'apprentissage de leurs élèves et puiser ensuite dans un large répertoire de savoirs théoriques et pratiques pour traiter ces problèmes. Les formations qui s'appuient sur ces processus d'enquête partent des pratiques concrètes pour asseoir les apprentissages, approfondir les contenus pédagogiques pertinents et les connaissances sur l'évaluation, tout en ancrant ces pratiques dans un processus d'enquête continu.

\section{Le renforcement des savoirs professionnels} par la pratique de l'enquête

Pour peser véritablement sur les résultats des élèves, les objectifs doivent être éclairés par une 
connaissance approfondie de l'hétérogénéité des élèves dans une situation de cours donnée. Le cycle d'enquête (voir l'annexe 2) commence donc par le recensement des besoins d'apprentissage des élèves par leurs enseignants. Pour explorer ces besoins, les enseignants doivent maîtriser les concepts de l'évaluation et disposer d'une banque d'outils à utiliser de manière souple selon le type de situation. Ainsi les cours sont davantage étayés par des faits solides et les enseignants sont plus réactifs aux besoins des élèves. La seconde partie du cycle porte sur l'identification des objectifs d'apprentissage professionnel des enseignants. Cet examen est beaucoup plus exigeant car il leur demande de réfléchir à leurs propres méthodes d'enseignement et de voir dans quelle mesure elles ont contribué aux acquis et à la réussite des élèves. Cette démarche exige une analyse plus collective qu'individuelle parce que la synthèse de la littérature internationale de recherche montre que la participation à une enquête mutuelle est une condition nécessaire (mais insuffisante) pour identifier les liens entre enseignement et apprentissage. Une fois ces liens identifiés, l'examen des besoins d'apprentissage des enseignants permet de promouvoir l'apprentissage des élèves. Un préalable pour l'enseignant est de se considérer comme agent du changement pour les élèves et leurs apprentissages. C'est une condition sine qua non de la corégulation ou de l'autorégulation de l'apprentissage professionnel. D'ailleurs, dans nombre d'études faisant état de gains durables, les enseignants ont d'autant plus le sentiment d'être efficaces que les résultats de leurs élèves s'améliorent.

Les activités de formation conçues pour approfondir les connaissances et affiner les compétences doivent être co-construites par les enseignants et les experts si l'objectif visé est bien l'autorégulation de l'apprentissage professionnel. Les résistances des enseignants sont levées s'ils voient la nécessité d'apprendre et s'ils ont un certain contrôle sur la démarche d'apprentissage. Ce qui est attendu en partie dans la formation professionnelle, c'est qu'elle amène des changements dans les pratiques de classe, sinon rien ne changera vraiment pour les élèves, pas plus qu'il n'y aura de changement notable de leurs résultats. Après avoir établi des objectifs d'apprentissage pour les enseignants et les élèves, puis mis en place des stratégies pédagogiques appropriées, il convient de suivre leurs effets sur les élèves car aucune pratique enseignante n'est assurée d'être efficace dans n'importe quel contexte. Cette méthode d'enquête nécessite donc certains outils ou ressources (séances d'observation, entretiens avec les élèves et analyse des progrès réalisés).

Dans les situations où les résultats ne montrent aucun signe d'amélioration, des ajustements supplémentaires s'avèrent nécessaires à un moment précis du processus. Ces ajustements peuvent concerner les objectifs, les séquences de formation ou leur mise en œuvre. Le processus d'enquête retourne donc aux acquis et aux besoins des élèves. La question est de savoir si les connaissances actuelles des élèves sont adaptées ou non à un enseignement efficace. La présence d'une personne extérieure au groupe - quelqu'un de l'encadrement ou situé hors de l'établissement - est donc nécessaire. Si les enseignants étaient en mesure de déterminer leurs besoins d'apprentissage sans un tel accompagnement, ils l'auraient déjà fait. Notons toutefois que des experts extérieurs ont aussi participé à des formations professionnelles inefficaces. Ce n'est donc pas leur présence qui fait la différence, mais la façon dont ils accompagnent l'apprentissage professionnel des enseignants.

\section{ILLUSTRATION À PARTIR D'UN CAS NÉO-ZÉLANDAIS}

Ce cas illustre un projet de développement professionnel continu, qui s'inscrivait dans une démarche nationale à l'initiative du ministère de l'Éducation néo-zélandais, pour prendre à bras-le-corps les difficultés scolaires des élèves les plus en échec. Depuis 2004, trois groupes d'écoles élémentaires ont pris part à ce projet de deux ans. Les élèves ont progressé bien au-delà des attentes, dans les trois cohortes, et notamment les élèves les plus en difficulté. Les gains moyens d'effet de taille de tous les élèves étaient de 1,2 en expression écrite et de 0,9 en lecture. Les gains étaient supérieurs pour les élèves situés parmi les $20 \%$ les plus faibles $(2,3$ en expression écrite ; 1,9 en lecture). Les gains annuels moyens attendus étaient de 0,20 en expression écrite et de 0,26 en lecture, gains confirmés la seconde année. Faute de place, seule la partie expression écrite du projet sera décrite ici, bien que la méthode de développement professionnel continu soit la même pour les deux champs curriculaires.

Dès l'origine du projet, priorité était donnée à l'amélioration des résultats des élèves. Le contrat passé entre le ministère de l'Éducation néozélandais et les prestataires extérieurs exigeait 
in fine l'amélioration de la réussite des élèves et elle passait par le respect de certaines conditions : un meilleur bagage universitaire et pédagogique des enseignants ; un meilleur transfert entre théorie et pratique pédagogique en littératie ; un encadrement plus efficace des communautés apprenantes. Les initiateurs du projet ont nommé 25 tuteurs auprès des établissements concernés et organisé des stages de développement professionnel sur le renforcement des contenus pédagogiques et évaluatifs, dans le cadre de processus d'enquête continus (voir l'annexe 2). La participation au projet se faisait sur la base du volontariat de chaque établissement, mais les enseignants n'avaient pas forcément leur mot à dire quant à leur participation éventuelle. Deux chercheurs en recherche formative ont suivi le projet, en endossant en même temps un rôle de consultant. Plusieurs études ont été entreprises : sur les pratiques de classe (Timperley \& Parr, 2009a), sur celles des tuteurs (Timperley, Parr \& Bertanees, 2009) et sur la chaîne d'influence s'étendant de la politique éducative à la pratique (Timperley \& Parr, 2009b).

Le projet a débuté par l'identification des besoins des élèves, à partir d'un outil d'évaluation permettant aux enseignants d'analyser la compréhension des structures profondes et de surface en expression écrite. Même en phase initiale, il est apparu nécessaire de préciser les besoins didactiques et pédagogiques des enseignants (voir l'annexe 2). Peu d'entre eux avaient réussi à noter correctement l'évaluation des élèves en expression écrite car ils n'étaient pas capables de faire une analyse fine des structures profondes. L'examen d'une situation d'apprentissage où trois enseignants d'un même établissement dispensaient des cours d'expression écrite à des fins de communication montre qu'ils s'efforçaient essentiellement de motiver les élèves lors de la rédaction d'un texte précis, mais que l'enseignement de l'expression écrite elle-même se limitait aux structures de surface (orthographe et grammaire). Sans surprise, lors des entretiens entre chercheurs et élèves, ces derniers indiquaient qu'ils ne savaient pas ce qu'ils étaient censés apprendre, hormis la ponctuation, l'orthographe, le respect du nombre de mots et la propreté de la copie. Suite à l'analyse de cette séance, les enseignants se sont rendu compte de leurs besoins réels d'apprentissage, notamment en termes de contenus pédagogiques.

Un examen plus poussé des besoins d'apprentissage professionnel des enseignants laissait apparaître un lien direct avec les besoins des élèves. Au moment même où les élèves étaient évalués, des données étaient recueillies sur les savoirs et les pratiques des enseignants. L'ensemble de ces outils a permis de dresser un tableau complet des influences mutuelles entre enseignement et apprentissage. Tous les établissements n'ont pas bénéficié de la même expertise ou n'ont pas souhaité déterminer leurs propres besoins d'apprentissage. Néanmoins, ils ont tous analysé d'abord le profil des élèves et identifié ceux qui réussissaient le moins bien, puis évalué ensuite leurs propres connaissances pédagogiques. Au départ, ils étaient peu nombreux à analyser l'expression écrite de leurs élèves en l'absence d'une aide extérieure. Le projet encourageait les enseignants à préparer et administrer des cours en phase avec les acquis et les besoins des élèves. Le renforcement des connaissances professionnelles des enseignants en constituait le préalable. Au cours de la seconde année du projet, lorsque les enseignants s'étaient familiarisés avec le processus d'enquête, chacun d'entre eux a mis sur pied des objectifs d'apprentissage avec les tuteurs à partir de l'analyse de ses propres besoins.

L'investigation de l'impact des nouvelles pratiques a commencé de façon informelle. Les enseignants ont été invités à interroger leurs élèves pour savoir s'ils comprenaient bien les objectifs d'apprentissage des cours. Le processus a été formalisé à la fin de la première année lorsque les élèves ont été réévalués avec le même outil d'évaluation. Les enseignants et l'encadrement ont pu, par la discussion sur les échantillons et la comparaison des résultats du début et de fin d'année, disséquer le type de progrès réalisé et formaliser le processus d'enquête concernant les résultats du changement de pratiques. Ils ont ainsi pu identifier l'efficacité des changements opérés dans leur façon d'enseigner. Un second cycle d'enquête sur les besoins d'apprentissage des élèves et des enseignants a vu le jour à partir des enseignements tirés du premier cycle d'enquête. Un processus itératif s'était donc mis en marche. À la suite de ces processus d'enquête entre les enseignants et leur hiérarchie, le besoin s'est fait sentir d'insister sur la méthode à utiliser. Les responsables politiques ont reconnu que, pour atteindre les objectifs fixés, il fallait plus d'un an, c'est-à-dire plus que la durée initialement retenue. Ils ont alors accordé des crédits supplémentaires pour couvrir les besoins financiers d'une seconde année. Les responsables du projet se sont aperçus, suite à des visites de terrain, que les connaissances pédagogiques des tuteurs étaient insuffisantes pour approfondir la méthode ; des stages ont donc été mis en place pour pallier cette lacune. Un besoin plus inattendu s'est fait sentir 
après l'observation du travail des tuteurs par les chercheurs dans les établissements : si certains avaient bien compris le mode de co-construction de la méthode, d'autres demeuraient plus repliés sur une interprétation individuelle du développement professionnel. Grâce à une série d'ateliers, dont un portait sur l'analyse de leurs interventions dans les établissements et était accompagné d'un questionnaire pour évaluer les réactions des enseignants à leurs interventions, les tuteurs ont gagné en assurance lors de la conduite des entretiens avec les enseignants.

\section{CONCLUSION}

Un développement professionnel de qualité est essentiel pour relever les défis de la réforme et améliorer les résultats des élèves les moins performants dans nos systèmes éducatifs. Les profils démographiques des élèves changent, de même que les connaissances sur les façons d'enseigner. Cet article a dressé un état des lieux des résultats issus d'une synthèse de la littérature internationale sur le type de développement professionnel propice à de meilleurs résultats scolaires et à un épanouissement des élèves sur les plans personnel et social. Nous avons montré que l'impact sur les apprentissages de nos élèves les plus défavorisés peut être accéléré par l'engagement des enseignants dans ce développement professionnel continu. La preuve par les résultats de l'efficacité du développement professionnel est un défi pour les chercheurs, les formateurs d'enseignants et les enseignants euxmêmes, concernant la méthode idoine permettant de renforcer les savoirs et l'offre de formation. L'évaluation de la formation professionnelle repose le plus souvent sur les réactions des enseignants à la fin de leur stage (Guskey, 2000). "Si nous acceptons l'idée que la finalité de l'école réside dans la formation des élèves et non des enseignants » (Camburn, 1997), alors l'efficacité de nos efforts doit être jugée à l'aune de l'amélioration des résultats des élèves.

L'argument de cet article selon lequel le développement professionnel peut s'avérer efficace va apparemment à l'encontre des réserves émises par certains praticiens, responsables politiques et chercheurs cités en début d'article, sur la capacité de la formation professionnelle à faire la différence en termes de résultats des élèves. Notre synthèse (Timperley, Wilson, Barrar et al., 2007) montre que ce n'est pas le développement professionnel en soi qui pose problème, mais plutôt la façon dont il est réalisé. Les formateurs doivent reconnaître la complexité des pratiques professionnelles et leur apporter de la valeur ajoutée, en accompagnant les enseignants dans la satisfaction des besoins d'un public hétérogène, notamment les élèves dont les résultats sont inférieurs à ceux des autres. II est indispensable d'avoir une vision globale des pratiques des enseignants plutôt que de les contourner, et d'apporter des propositions et des pratiques alternatives par des exemples concrets. Les enseignants ont besoin d'une offre de contenus pédagogiques pertinente et d'un enrichissement de leurs connaissances professionnelles sur l'évaluation, par un mélange de théorie et de pratique. Si on enlève n'importe lequel de ces éléments, l'impact a toutes les chances d'être fortement diminué. Une enquête systématique éclairée par des sources fiables est au cœur de tout processus de développement professionnel continu. Si les enseignants refusent de s'engager dans ce processus, ils n'ont alors guère d'informations sur ce qu'ils doivent apprendre et ce qu'ils peuvent faire pour améliorer les résultats des élèves ; ils ne savent pas non plus si les changements qu'ils opèrent ont l'effet désiré. Le préalable à la réussite de ce processus est que les enseignants soient convaincus que leur propre formation relève de leur responsabilité professionnelle, y compris le souci de vérifier l'efficacité de leurs pratiques sur les résultats des élèves. Ainsi des cycles renouvelés d'enquête, de renforcement des capacités et d'amélioration de leurs pratiques deviennent partie intégrante de leur quotidien.

Helen Timperley h.timperley@auckland.ac.nz School of Teaching, Learning and Development, University of Auckland, Nouvelle-Zélande

\section{NOTE}

1 La taille d'effet (traduction de l'anglais effect size) est un indice statistique qui permet de quantifier l'amplitude de l'effet d'un traitement que l'on a évalué et qui reflète le gain éventuel du groupe qui a bénéficié de ce traitement par rapport au groupe témoin. 


\section{BIBLIOGRAPHIE}

BAKER S. \& SMITH S. (1999). " Starting off on the right foot: The influence of four principles of professional development in improving literacy instruction in two kindergarten programs ». Learning Disabilities Research \& Practice, vol. 14, $\mathrm{n}^{\circ}$ 4, p. 239-253.

BORMAN G., SLAVIN R., CHEUNG A. et al. (2005). " Success for all: First-year results from the national randomized field trial ". Educational Evaluation and Policy Analysis, vol. $27, \mathrm{n}^{\circ} 1$, p. 1-22.

CAMBURN E. (1997). The impact of professional community on teacher learning and instructional practice. Thèse de doctorat, sciences de l'éducation, University of Chicago, Chicago.

CARPENTER T., FENNEMA E., PETERSON P. et al. (1989). "Using knowledge of children's mathematics thinking in classroom teaching: An experimental study ». American Educational Research Journal, vol. 26, n 4, p. 499-553.

COHEN J. (1988). Statistical power analysis for the behavioral sciences. Hillsdale : Erlbaum.

CUTTANCE P. (1998). "Quality assurance reviews as a catalyst for school improvement in Australia ". In A. Hargreaves, A. Lieberman, M. Fullan \& D. Hopkins (dir.), International handbook of educational change, vol. 5. Dordrecht : Kluwer Publishers, p. 1135-1162.

DATNOW A., BORMAN G., STRINGFIELD S. et al. (2003). " Comprehensive school reform in culturally and linguistically diverse contexts: Implementation and outcomes from a four-year study ". Educational Evaluation and Policy Analysis, vol. 25, n 2, p. 143-170.

DONOVAN M., BRANSFORD J. \& PELLEGRINO J. (1999). How people learn. Bridging research and practice. Washington : National Academy Press.

FISHMAN B., MARX R., BESTA S. \& TAL R. (2003). « Linking teacher and student learning to improve professional development in systemic reform ». Teaching and Teacher Education, vol. $19, n^{\circ}$ 6, p. 643-658.

GUSKEY T. (2000). Evaluating professional development. Thousand Oaks : Corwin Press.

HATTIE J. (1990). "Performance indicators in education ». Australian Journal of Education, vol. 34, n 3, p. 249-276.

HATTIE J. (1992). "Measuring the effects of schooling". Australian Journal of Education, vol. $36, \mathrm{n}^{\circ}$ 1, p. 5-13.

LIPMAN P. (1997). « Restructuring in context: A case study of teacher participation and the dynamics of ideology, race and power ". American Educational Research Journal, vol. $34, n^{\circ} 1$, p. 3-37.

LIPSEY M. \& WILSON D. (1993). " The efficacy of psychological, educational, and behavioral treatment: Confirmation from meta-analysis ". American Psychologist, vol. 48, n 12, p. 1181-1209.

McNAUGHTON S., LAI M., MacDONALD S. \& FARRY S. (2004). " Designing more effective teaching of comprehension in culturally and linguistically diverse classrooms in New Zealand ». Australian Journal of Language and Literacy, vol. 27, $\mathrm{n}^{\circ}$ 3, p. 184-197.
MUIJS D. \& REYNOLDS D. (2001). Effective teaching. Evidence and practice. Londres : Paul Chapman Publishing.

NATIONAL STAFF DEVELOPMENT COUNCIL (2001). National staff development council's standards for staff development. Oxford : National Staff Development Council.

NYE B., KONSTANTANOPOULOS S. \& HEDGES L. (2004). " How large are teacher effects? " Educational Evaluation and Policy Analysis, vol. 26, n 3, p. 237-257.

ROBBINS P. \& WOLFE P. (1987). « Reflections on a Hunterbased staff development project ". Educational Leadership, vol. 44, n 5, p. 56-61.

SAXE G., GEARHART M. \& NASIR N. (2001). " Enhancing students' understanding of mathematics: A study of three contrasting approaches to professional support ». Journal of Mathematics Teacher Education, vol. 4, $\mathrm{n}^{\circ}$, p. 55-79.

SHULMAN L. (1986). "Those who understand: A conception of teacher knowledge ". American Educator, vol. 10, $\mathrm{n}^{\circ} 1$, p. 9-15.

SHULMAN L. (1987). « Knowledge and teaching: Foundations of the new reform ». Harvard Educational Review, vol. 57, $\mathrm{n}^{\circ} 1$, p. 1-22.

SIJDE P. van der (1989). «The effect of a brief teacher training on student achievement ". Teaching and Teacher Education, vol. 5, $\mathrm{n}^{\circ}$ 4, p. 303-314.

SPARKS D. (2004). " Focusing staff development on improving the learning of all students ». In G. Cawelti (dir.), Handbook of research on improving student achievement. Arlington : Educational Research Service.

STALLINGS J. \& KRASAVAGE E. (1986). « Program implementation and student achievement in a four-year Madeline Hunter follow-through project ". The Elementary School Journal, vol. 87, n 2, p. 117-137.

TALLMADGE G. (1977). The joint dissemination review panel idea book. Washington : National Institute of Education.

TIMPERLEY H. \& PARR J. (2009a). "What is this lesson about? Instructional processes and student understandings in writing classrooms ". The Curriculum Journal, vol. $20, n^{\circ} 1$, p. 43-60.

TIMPERLEY H. \& PARR J. (2009b). "The chain of influence from policy to practice in the New Zealand literacy strategy ». Research Papers in Education, vol. 24, $\mathrm{n}^{\circ} 2$, p. $135-154$.

TIMPERLEY H., PARR J. \& BERTANEES C. (2009). «Promoting professional inquiry for improved outcomes for students in New Zealand ". Professional Development in Education, vol. 35, $\mathrm{n}^{\circ}$ 2, p. 227-245.

TIMPERLEY H., WILSON A., BARRAR H. \& FUNG I. (2007). Teacher professional learning and development: Best evidence synthesis iteration (BES). Wellington : Ministry of Education. Disponible sur Internet à l'adresse : <http://www.educationcounts.govt.nz/publications/ series/2515/15341> (consulté le 26 janvier 2011). 


\section{ANNEXE 1. CADRE D'ANALYSE DE L'EFFICACITÉ DES FORMATIONS PROFESSIONNELLES}

\author{
Contexte socioculturel au sens large \\ - politiques éducatives formelles ; \\ - discours dominants ; \\ - organisation scolaire.
}

\section{Contexte du développement professionnel}

- personnes et détails pratiques

- objectifs du développement professionnel.

\section{Contenu des dispositifs de formation professionnelle}

Les interprétations conceptuelles et l'approfondissement des compétences à travers les activités de formation professionnelle portaient sur :

- les savoirs disciplinaires ou curriculaires ;

- les savoirs pédagogiques ;

- l'évaluation:

- les élèves (apprentissages, comportement, culture) ;

- la socialisation des élèves dans les établissements ;

- les ressources culturelles et linguistiques ;

- l'impact des pratiques enseignantes sur les élèves dans leur diversité.

\section{Activités au service des apprentissages}

Les activités de développement professionnel conçues pour approfondir les compétences professionnelles portaient sur:

- écouter/observer;

- être observé et bénéficier du retour d'expérience ;

- entamer des lectures professionnelles ;

- partager ses pratiques et leurs conséquences ;

- analyser la compréhension des élèves et leurs résultats :

- analyser ses pratiques actuelles au regard de nouvelles pratiques

- débattre d'enjeux séparément ou mutuellement identifiés.

\section{Processus d'apprentissage (itératifs)}

Les processus d'apprentissage visant l'acquisition ou l'approfondissement des compétences impliquent au moins un des processus cycliques suivants :

\begin{tabular}{|c|c|}
\hline \multirow[b]{2}{*}{ Processus 1} & S'appuyer sur les savoirs existants \\
\hline & $\begin{array}{l}\text { Résultat : consolidation et/ou analyse } \\
\text { des savoirs existants }\end{array}$ \\
\hline \multirow[t]{2}{*}{ Processus 2} & $\begin{array}{l}\text { Se familiariser avec les nouvelles } \\
\text { informations/compétences et les } \\
\text { intégrer à son système de valeurs et de } \\
\text { croyances actuel }\end{array}$ \\
\hline & $\begin{array}{l}\text { Résultat : adoption ou adaptation des } \\
\text { nouveaux savoirs }\end{array}$ \\
\hline \multirow[b]{2}{*}{ Processus 3} & $\begin{array}{l}\text { Créer une dissonance par rapport à sa } \\
\text { position actuelle (valeurs et croyances) }\end{array}$ \\
\hline & $\begin{array}{l}\text { Résultat : repositionnement et } \\
\text { reconstruction du système de croyances } \\
\text { et de valeurs }\end{array}$ \\
\hline \multirow{2}{*}{ Processus 4} & $\begin{array}{l}\text { Amélioration de l'autorégulation et de la } \\
\text { corégulation }\end{array}$ \\
\hline & $\begin{array}{l}\text { Résultat : adoption des cycles } \\
\text { d'enquête }\end{array}$ \\
\hline
\end{tabular}

\section{Réactions des enseignants et des élèves}

Les enseignants et les élèves font au moins une des choses suivantes:

- rejettent ou ignorent les nouvelles théories et pratiques et reprennent leurs pratiques précédentes ;

- reprennent leurs pratiques précédentes croyant qu'il s'agit de nouvelles pratiques ;

- choisissent certaines parties des nouvelles théories et pratiques et les adaptent à leurs pratiques actuelles ;

- les appliquent comme prévu ;

- s'approprient et appliquent les nouvelles théories et modifient leurs pratiques de façon significative.

\section{Impact sur les élèves}

Effets de la formation professionnelle sur les items "réussite », « attitude », « identité sociale»:

- aucune amélioration (voire baisse) des résultats ;

- amélioration légère d'un nombre de paramètres restreints ;

- amélioration significative d'un grand nombre de paramètres. 
ANNEXE 2. CYCLES D'ENQUÊTE AU SERVICE DU RENFORCEMENT DES COMPÉTENCES DES ENSEIGNANTS

De quelles connaissances et compétences nos élèves ont-ils besoin ?

- Quels sont leurs acquis?

- Quelles sources sont utilisées pour vérifier ces acquis?

- Que doivent-ils apprendre et faire ?

- Comment s'appuyer sur leurs acquis ?

De quelles connaissances et compétences avons-nous besoin en tant que professionnels ?

- De quelle manière avons-nous contribué aux résultats actuels des élèves ?

- De quelles ressources immédiatement mobilisables disposons-nous pour améliorer leurs résultats?

- Quelles sont les compétences à acquérir pour améliorer leurs résultats ?
- Quelles sources étayées pouvons-nous utiliser?

Approfondir les savoirs professionnels et mettre à jour ses compétences par davantage de formation professionnelle

\section{Mobiliser les élèves autour de nouvelles} expériences d'apprentissage

Quel est l'impact de ces changements ?

Quelle est l'efficacité des contenus de formation professionnelle sur les apprentissages et le bien-être des élèves ? 